\title{
Electron-Optical Bench
}

\section{Marton, M. M. Morgan, D. C. Schubert, J. R. Shah, and J. A. Simpson}

\begin{abstract}
A versatile electron-optical bench has been constructed for the extensive study of electron-optical elements. Three carriages for magnetic lenses and four holders for apertures, objectives, and meshes are arranged appropriately in the vacuum chamber, while provision is made for external positioning with 3 degrees of freedom for each element. The design and experimental technique are discussed in detail. A set of measurements of the focal length of a magnetic lens is presented to illustrate the practical employment of the bench and to indicate the limits of accuracy that can be attained.
\end{abstract}

\section{Introduction}

For the extensive study of the properties of electron-optical elements, an electron-optical bench has been constructed. This bench provides for the control of the positions of such elements from outside the vacuum system and quantitative measurement of the image formed on a fluorescent screen.

Very few attempts have been made to design an electron-optical bench. Among the very few electron optical benches, which have been built in the past, is the instrument designed by Reisner and Picard. ${ }^{1}$ A minor feature of that bench was the provision for visual observation of the parts inside the vacuum chamber.

Inasmuch as all measurements required during the operation of an electron-optical bench can be made externally, the ability to see the optical system need not be considered particularly useful. For simplicity of construction and protection against X-rays the NBS electron-optical bench was made with an allmetal cylindrical vacuum chamber. It was designed to satisfy the following requirements: 1 . The bench should accommodate three carriages for lenses with maximum diameter of $7 \mathrm{in}$. and four holders for apertures, test objects, and meshes (used for the electron-optical shadow method). ${ }^{2}$

2. A movement of $10 \mathrm{in}$. along the axis of the bench and $1 / 2$ in. radially should be possible for each component of the system without breaking the vacuum.

\section{Description of Apparatus}

\subsection{Vacuum Chamber}

A rolled-steel cylinder of 15-in. inner diameter, $1 / 4$-in. wall and 39 -in. length is part of the vacuum chamber used to house the electron-optical system. The end plate supporting the gun is permanently fixed to the cylinder with bolts, the vacuum seal being provided by a rubber gasket. A dural plate, A, figure 1 , on wheels serves as a bed on which the electron-optical system is mounted. The specimen and aperture mounts, B, and lens carriages, C, slide in a groove along the bed. The bed may be rolled into or out of the chamber. One end of the bed is

J. H. Reisner and R. G. Picard, Rev. Sci, Instr. 19, 556 (1948).

2 L. Marton and S. H. Lachenbruch, J. Applied Phys, 20, 1171 (1949); J. Research NBS 43, 409 (1949). attached to the face plate by means of a flexible hinge, which does not encounter the strain that might result if a rigid joint were used.

The face plate seals against the end of the tube by a neoprene gasket compressed by atmospheric pressure. The fluorescent screen is coated on a glass plate $\left(5 \frac{7}{8} \mathrm{in}\right.$. in diameter and $7 / 8$-in. thick) in the face plate. Motion of the elements is controlled by means of connecting shafts passing through "Wilson seals," $\mathrm{E}$, in the face plate.

\subsection{Lens Carriages}

The radial adjustment of each lens is obtained by properly combining controlled vertical and horizontal motions of the lens carriage. For vertical adjustment a double-threaded worm, A, figure 2, attached to a connecting shaft, B, engages a gear, $\mathrm{C}$, the shaft of which rotates in the bottom dural plate, D. A screw pinned to the worm gear turns in the top plate, $\mathrm{E}$, thus moving it vertically when the worm is rotated. The top plate is constrained in its motion by sliding on four pins, F, attached to the lower plate. Horizontal adjustment is similarly achieved by a worm and gear arrangement sliding the plate, $\mathrm{G}$, over plate, $\mathrm{E}$, the motion being constrained by a tongue in groove. Since the vertical position of the worm, $\mathrm{H}$, changes, two universal joints are inserted in its connecting shaft. The axial position of the carriage is controlled by pushing or pulling the connecting shafts through the Wilson seals.

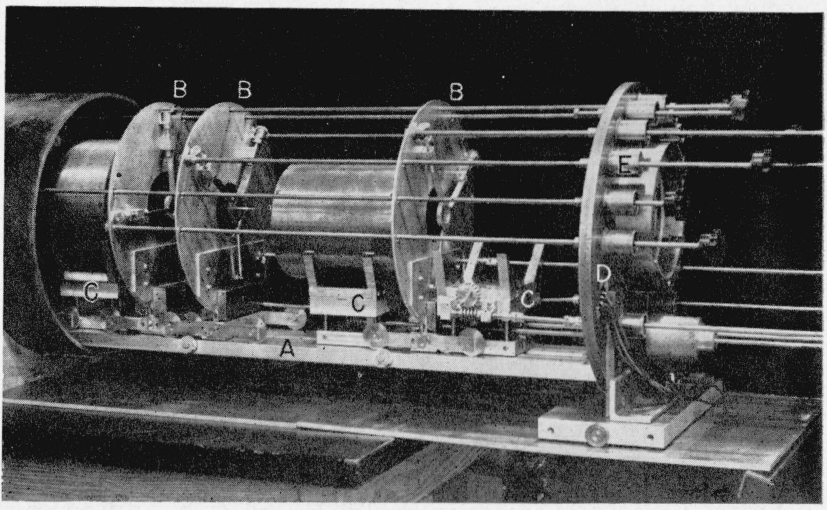

FIGURE 1. Vacuum chamber of electron-optical bench open for changing elements. 


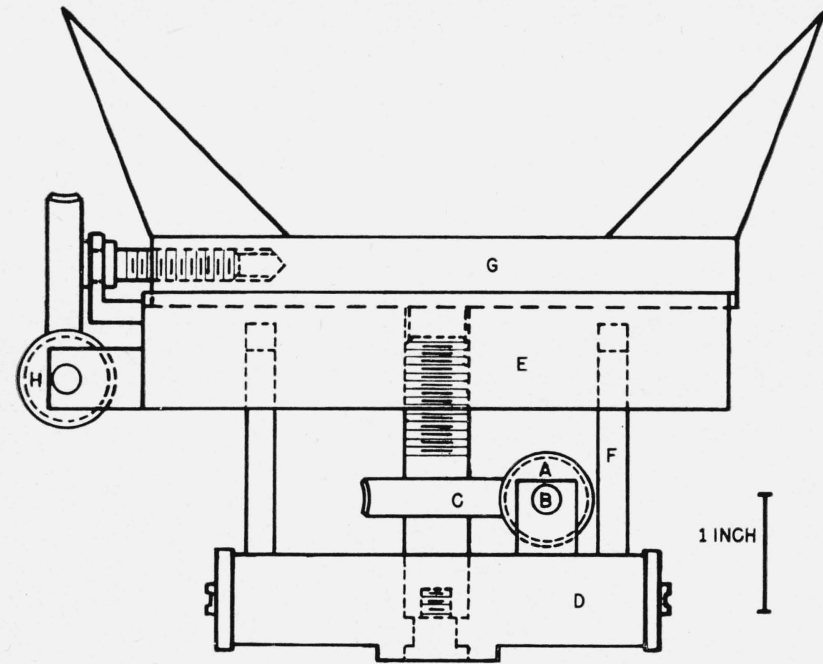

FIGURE 2. Lens carriage for electron-optical bench.

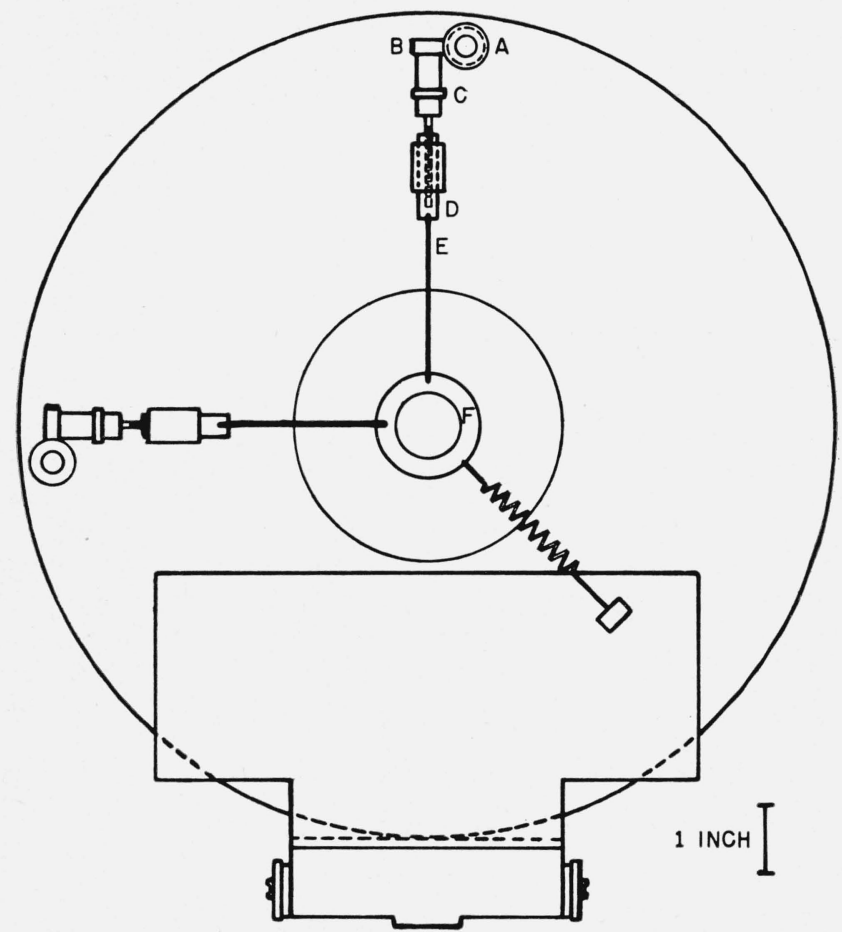

FIGURE 3. Specimen and aperture mount for electron-optical

\subsection{Specimen, Mesh, and Aperture Mounts}

If one is to achieve a smooth motion of specimens or test objects with a minimum of jerkiness, the drive must be made as nearly frictionless as possible. Pursuant to this requirement, the radial motion in the present arrangement is constrained by elastic deformation of phosphor bronze strips instead of sliding surfaces. A similar arrangement for positioning an electron microscope stage, which reduced friction to an even greater degree, was described in an earlier paper. ${ }^{3}$

\footnotetext{
${ }^{3}$ L. Marton, J. Applied Phys. 16, 131, 1945.
}

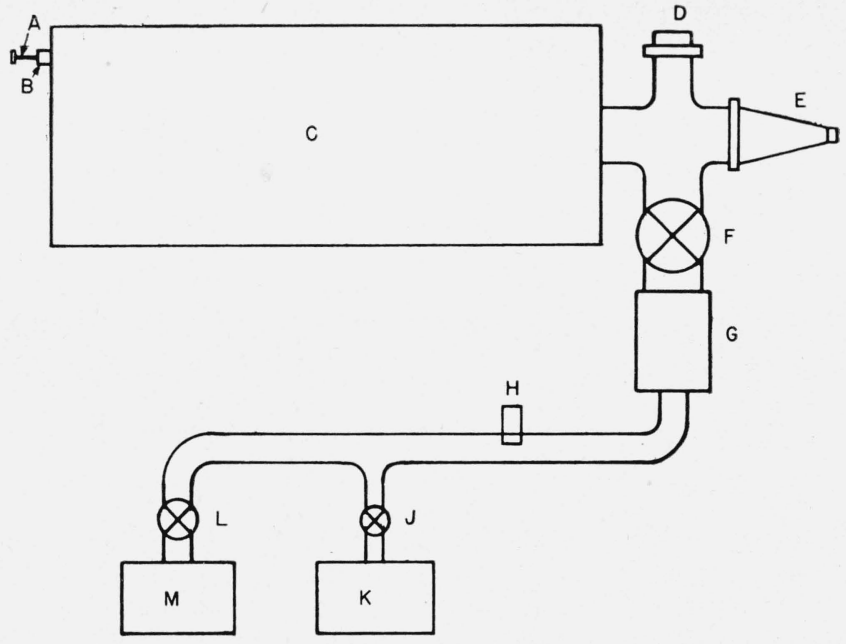

FiguRE 4. Vacuum system for electron-optical bench.

A, Air bleeder; B, Wilson seal; C, vacuum chamber; D, ionization gage; E, electron gun; F, 4-in. main valve; G, 4-in. diffusion pump; H, thermocouple gage; J, 1-in. 3-way valve; K, duo-seal pump; L, 2-in. valve; M, Kinney pump.

Radial adjustment of the specimen, mesh, or aperture holders is provided by a pair of worm and gear mechanisms at right angles to each other. A worm, A, figure 3 , attached at the end of a connecting shaft engages a gear, B, rotating in a collar, C, fixed to the mount. A screw, pinned at the end of the worm gear shaft, turns in a sleeve, D, which is attached by means of a flexible phosphor-bronze strip, E, to the ring, $\mathrm{F}$, into which an appropriate specimen, mesh, or aperture holder is screwed. In this way the ring is moved along the axis of the worm gear, B,. The elastic deformation of the phosphor-bronze connection, because of its orientation, provides a simple solution of the mechanical linkage problem of moving the ring, without the use of friction devices, in a plane perpendicular to the axis of the bench while maintaining rigidity in the axial direction. Because of the arrangement of the Wilson seals, the connecting shafts had to be attached to the circular plate forming the body of the mount near its top, thus leading to somewhat jerky axial motion. This jerkiness was reduced by using a long, heavily weighted wheel base for the mount.

\subsection{Vacuum System}

A schematic diagram of the vacuum system is shown in figure 4 . The lens currents are led through the vacuum wall by metal-glass seals in the face plate; and the conductors are insulated by ceramic beads, which produce less outgassing difficulties than would result if rubber or plastic insulation were used. This is realized by means of a large fore-vacuum pump and a 4-in. oil diffusion pump. This large pump brings the pressure down from the atmospheric to about 10 microns of mercury in 5 minutes. However, as this pump causes considerable vibrations, it is turned off after initial pumping and the quieter small fore-vacuum pump is used during final adjustments and measurements. The normal operat- 
ing pressure is about $3 \times 10^{-2}$ micron of mercury, measured by an ion gauge near the gun.

The special three-way valve near the small capacity pump is constructed to bleed air into the vacuum system at a controlled rate, as well as to insert the small capacity pump into or isolate it from the system.

\subsection{Electrical System}

A conventional 100-kilovolt X-ray power supply unit, consisting of a voltage doubler circuit with two 50-kilovolt rectifier tubes, is used as the highvoltage supply. Stabilization is provided by two 0.1 -micro-farad condensers in parallel with the circuit. The voltage is measured by the current passing through a 1,000 -megohm bleeder resistance connected across the high-voltage supply. The normal range of operation is from 20 to 70 kilovolts. The filament is heated by a high-frequency power supply. The lens current power supplies are a modification of those described in an earlier paper (see footnote 3) and give up to 500 milliamperes with a stability of better than 1 part in 10,000 .

The lenses available for use in the bench were originally designed for use with the coils out in the atmosphere rather than in a vacuum chamber; consequently they were poorly suited to operation within the bench, and the coils released large quantities of gas to the vacuum system. An extremely rapid pumping system was needed to maintain a working vacuum under such unfavorale conditions.

\subsection{Optical System}

A great portion of the use of an electron-optical bench is in the empirical approach to the development of electron-optical lens systems. In such work the result of one measurement often determines what conditions should be used for the next measurement. Hence the direct method of measuring the image is preferred as quicker, simpler, and cheaper than the photographic method of recording results. A low-power traveling telescope reading 0.001-in. with a total travel of $2 \frac{3}{4}$-in. was specially built for measurement of the image on the fluorescent screen. Since the bench was designed for use with magnetic lenses with their attendant rotation of the image, the measuring telescope is mounted on a turntable, which can be moved either horizontally or vertically a distance of 3 in. in such a way that its center of rotation may be concentric with that of the image. In this way all measurements are radial. Variable intensity side illumination of the reticule reduces the difficulty in seeing the cross hair when the intensity of the pattern on the screen is low.

Zinc cadmium silicate was used for the fluorescent screen. To make the screen conducting, it was aluminized, using the technique suggested by Bachman. ${ }^{4}$

A $100-\mathrm{kv}$ electron gun, identical to the one described in an earlier publication (see footnote 3 ), is

${ }^{4}$ C. H. Bachman, Techniques in experimental electronics, p. 235 (John Wiley \& Sons, Inc., New York, N. Y., 1948). the source of electrons, the grid bias being obtained from a potentiometer connected across three 45 -volt batteries in series.

\subsection{Measurements}

Sample measurements taken on the electronoptical bench are presented graphically (fig. 5) for the purpose of illustrating its use. These measurements of focal length were taken with the condenser lens, a mesh object, and the objective lens in the system. The objective lens has 10,000 turns. A symmetrical cold-rolled steel pole piece of 0.081-in. bore diameter and 0.060 -in. gap width fits into the $5 / 8$-in. inner diameter of the iron enclosure of the lens. Diffuse illumination of the object, obtained by applying a thin Formvar film and a thin conducting aluminum coating on the source side of the mesh was used to make focusing critical. The image distance was held constant throughout the measurements; for a particular accelerating voltage the object position was adjusted to give approximate focus for different objective currents, the final focus being made by a small adjustment of the current. The focal length was computed by the equation $f=q /(M+1)$, where $q$ is the image distance and $M$ is the magnification.

With the assumption that the curves drawn are approximately as good fits as would be obtained by

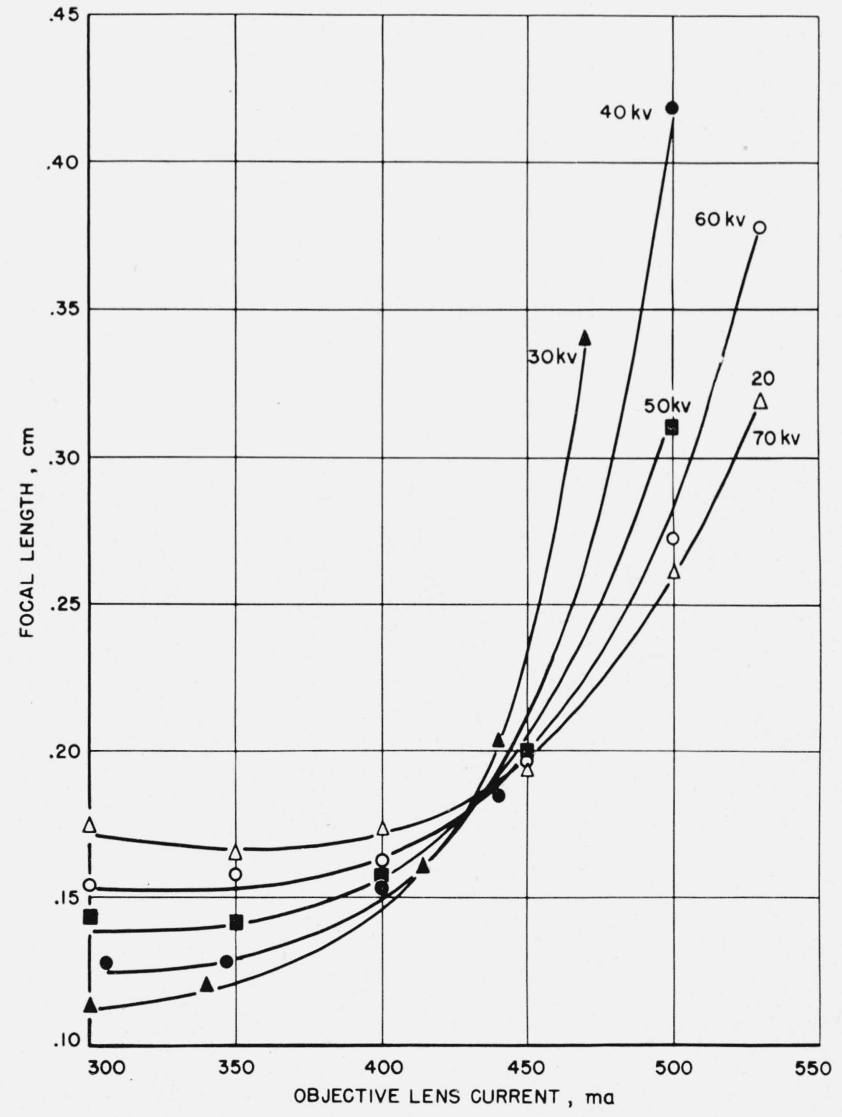

FIGURE 5. Variation of focal length of objective lens with lens current for different accelerating voltages. 
applying the least-squares method to a "known" function, the rms error in the focal length is found to be less than $\pm 0.001 \mathrm{~cm}$. From the graph the maximum error seems about $\pm 0.01 \mathrm{~cm}$. The major limitation on accuracy seems to be the difficulty in setting the cross hair on a point of the mesh image. Improvement of the cross-hair design and development of greater skill in setting may reduce the error from this source to less than 1 percent from a present value as high as 2.5 percent. Another limitation is in the precision with which the image can be focused. With the objective lens and pole piece previously described, a change of lens current of the order of \pm 3 ma, over the range of currents used, is required to produce a detectable change in definition from best focus, that is, the image appears to be in perfect focus over a range of about $6 \mathrm{ma}$. The center of this range can be located within about \pm 2 ma. It is believed that this cannot be much improved without elaborate additions to the equipment. Errors due to small inaccuracies in alinement and to hysteresis are believed to be negligible compared to these two limiting factors; no particular care was taken to eliminate hysteresis errors in these preliminary measurements.

The electron-optical bench is quite versatile because of the ease with which elements may be added and removed, and because three-dimensional movement of all elements is possible from outside the vacuum wall. It can be used for almost any electronoptical measurement and can be adapted for use in electron-optical field mapping and as an electron microscope.

Practically all the construction work was done by August B. Dauses, whose collaboration is gratefully acknowledged. Two Grant-in-Aids, one from Sigma $\mathrm{Xi}$ Research Fund and one from the American Philosophical Society, are gratefully acknowledged by J. R. Shah. These enabled Mr. Shah to extend a stay in the United States by several months and thus contribute to the completion of this project.

Washington, April 16, 1951. 